\title{
Journey from solitons to nanophotonics: an interview with Professor Yuri Kivshar
}

\author{
Guoqing Chang* \\ Institute of Physics, Chinese Academy of Sciences, China
}

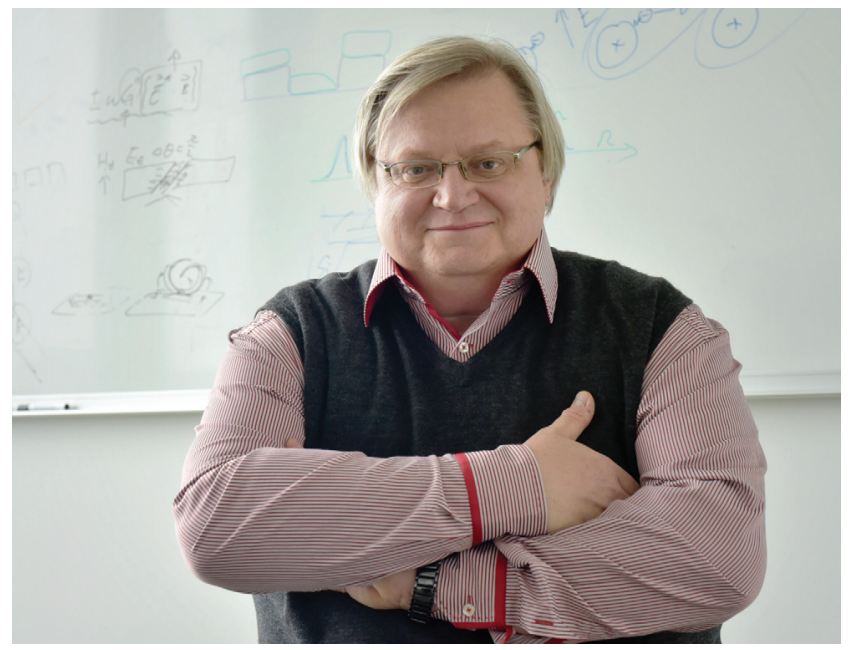

Professor Yuri Kivshar, Australian National University, Australia

Guoqing Chang: What inspired your interest in nonlinear physics? Yuri Kivshar: I was born in the city of Kharkov and studied physics at the Kharkov National University where Soviet academician Lev Landau was Chair of the Department of Theoretical Physics. I liked math, and even considered for some time being a mathematician. But I discovered that true mathematicians must prove theorems, and I did not like theorems. I became interested in physics because physicists study real things. And theoretical physics looked more attractive because it combines both math and physics.

My $\mathrm{PhD}$ supervisor was academician Arnold Kosevich, who is the silent co-author of the seventh volume of Landau-Lifshitz Course of Theoretical Physics; rumor says he helped to write a large part of the book Theory of Elasticity.

At the beginning of the fourth year (or maybe even at the end of the third year) at the University, I got involved in research work. When I approached Professor Kosevich, he was working on the FrenkelKontorova model which is one of the simplest models of crystal dislocation, known to reduce to the sine-Gordon equation with soliton solutions.

I was fascinated by the properties of solitons because they correspond to nice solutions of nonlinear partial differential equations and might appear in realistic experiments. Besides providing exact solutions, the physics behind solitons involves self-induced energy localization and competition between dispersion (or diffraction) and nonlinearity.

*Address all correspondence to Guoqing Chang, guoqing.chang@iphy.ac.cn (C) The Authors. Published by SPIE and CLP under a Creative Commons Attribution 4.0 Unported License. Distribution or reproduction of this work in whole or in part requires full attribution of the original publication, including its DOI. [DOI: 10.1117/1 .AP.3.1.010502]
Guoqing Chang: When did you first hear about nonlinear optics? Yuri Kivshar: My course had about 100 students who were associated with different departments, including Department of Theoretical Physics and Department of Optics. I was in the Department of Theoretical Physics, and I did not know nonlinear optics at all when I started my research on solitons with Professor Kosevich. It had nothing to do with optics, and we never discussed optics.

Once I went for lunch with some of my classmates from the Department of Optics. One of them told me his research in optics was boring. I asked him why he went there in the first place. He told me that he initially expected to work on nonlinear optics. That was my first time to hear of nonlinear optics. I asked him what nonlinear optics is and he said, "It's about nonlinear effects such as frequency generation, all this nice stuff." And I thought, "Wow, it's probably what may be related to solitons."

Guoqing Chang: How did you move into the soliton research in the context of nonlinear optics?

Yuri Kivshar: Solid state physics is a difficult field because it is not linked to simple equations. In contrast, we have Maxwell's equations in optics; even its simplified form of the nonlinear Schrödinger equation works very well. In solid state physics, we have heating, noise, different types of multi-phonon excitations, and a lot of statistics, which make it impossible to generate any clear theory. In general, it is very hard to make a link between what you're doing with theory and what you measure in an experiment. That made it completely boring. I had developed dissatisfaction in my research, as I wanted something more exciting.

In 1989, I met a few people from Moscow who were working on optical fibers with Professor Evgeni Dianov at the Prokhorov Institute of General Physics. After some discussions, we realized that our work on solitons might be useful for fiber optics, and we started to study specific properties of solitons described by the nonlinear equations in fibers. That is how optical fiber solitons came to play, and that is how I was attracted to soliton research under the context of nonlinear optics.

Guoqing Chang: Around every 8 years, you have moved into a new field.

Yuri Kivshar: My philosophy is that you should change your field of research every 8-10 years if you want to remain active in science. I roughly followed that path during my research career having what I call "four lives in science"-solitons, photonic crystals, metamaterials, and now nanophotonics. My switch to a different field seemed to be triggered by some lucky events. I was very lucky to meet with many great people in my life and almost each of these meetings have influenced my decision on choosing a fresh research direction.

After receiving a $\mathrm{PhD}$ degree, I worked at the Institute for Low Temperature Physics for a couple of years. I left the Soviet Union first to Spain in 1989, and then moved from Spain to Germany as a Humboldt Fellow. Later, I was invited by Allan Snyder who co- 
authored the famous book Optical Waveguide Theory. Snyder was working on spatial solitons and I already had a lot of knowledge of optical solitons in optical fibers. These are temporal solitons, and complicated effects (e.g., Raman scattering and high-order dispersion) need to be considered when a soliton becomes too short in duration. But spatial solitons are simpler, so then I started to study them. Soon, I extended my spatial soliton research into the context of discrete systems and optical lattices.

Since 1993, I have worked at the Australian National University. Professor Costas Soukoulis once visited me in Australia and introduced me to the field of photonic crystals. I immediately realized that it is a great field for nonlinear optics where my old experience in discrete systems can be useful, and that is how I switched to nonlinear photonic crystals.

It was also a very interesting story about how I came to metamaterials. I was an editor of Physical Review E and was visiting the US several times a year. At the APS March conference-more than 15 years ago, we had the editorial meetings and I met again with Professor Costas Soukoulis. He told me that someone claimed that refractive index can be negative, but he did not believe it. He grabbed me in the corridor and took me to David Smith's talk on negative refractive index he measured in microwaves - that is how I learned about metamaterials. I experienced a lot of excitement and started to expand my research into this field. I have a broad research background which is helpful for my moving into new research fields.

Guoqing Chang: Recently you are working in an emerging fieldMie-tronics. Could you give a brief introduction about this field?

Yuri Kivshar: Mie-tronics is photonics driven by Mie resonators. These resonators exhibit large permittivity (i.e., epsilon) and thus support magnetic dipole resonances. The conventional design of metamaterials relies on plasmonic structures. Mie resonators are larger than plasmonics in size, but they have both electric and magnetic responses. When constructing metamaterials, we used to apply homogenization by field averaging, but this is unnecessary when using Mie resonators. You may apply these responses for simple dipole and multipoles, and construct different types of radiation patterns, or couple different resonators (e.g., quantum resonators, meta-surfaces, etc.) to achieve collective resonances. Plasmonic structures have a small $Q$ factor of about 10. The $Q$ factor of dielectric micro-ring resonator is $10^{5}$ while Mieresonant particles may have a $Q$ factor of about 200 achieved by a smart engineering of bound states in the continuum, and this can be boosted in arrays up to $10^{5}$ with footprints much smaller than those of the micro-ring systems. The radiation, transmission, and many other properties of metamaterials made of Mie resonators can be flexibly controlled, which is uncovered by interesting physics with new possibilities. Since no metal is used, loss can be avoided as well.

Guoqing Chang: How did you start to work on Mie-tronics?

Yuri Kivshar: My friend Boris Luk'yanchuk studied the Mie resonances for many years. I did read his theory paper published on Physical Review $B$ and realized the potential of Mie resonators in developing metamaterials. There were two big streams in metamaterial research: one was replacing plasmonic resonances by Mie resonances (or something similar) and the other was homogenization. Boris Luk'yanchuk and I had tremendous discussions, and we realized later that Mie resonators supporting not only electric but also magnetic multipoles may be unique elements for "meta" research because we can interfere different multipoles and achieve novel functionalities such as unidirectional scattering associated with the Kerker effect. So, this field exhibits completely different potential.
Guoqing Chang: What triggered your investigation on the bound state in the continuum?

Yuri Kivshar: The idea of the bound state in the continuum came truly unexpected. It came from an interesting experiment: we tried to study what will be the transition between metamaterials and photonic crystals. That is not easy because many structures at different scales need to be measured. With my colleagues at the ITMO University in Russia, we carried out experiments using very simple structures. We took tubes and poured water and created a periodic structure from tubes of water. The permittivity of water is huge and varies between 70 and 90 with temperature. So, we can easily tune it by heating the water, and change the structure period as well. We carried out an experiment, and the results were published in Nature Communications. Very funny experiment. It is a big macroscopic photonic crystal we have shown in the photos.

We then asked the question: what happens for a single water tube? A single water tube behaves like a high-index resonator. When we look at the water tube, a student put water differently in different experiments, and we found that for different levels of water the scattering diagram becomes completely different. Then we mapped everything on a kind of dispersion curve for the aspect ratio and revealed a signature of strong coupling. That is how we uncovered the bound state in the continuum, and one of the scenarios which appears through strong coupling. It was a lot of fun. In 2019, we published a highly cited paper in Advanced Photonics (by now cited 81 times), and later published the experimental results for optics in Science where we observed record-high secondharmonic generation from this type of resonators due to the bound state in the continuum.

Guoqing Chang: Mie resonators also give rise to novel nonlinear optical phenomena, right?

Yuri Kivshar: Nonlinear optics in our field is different from that in crystals or gases. The bound state in the continuum allows a very high energy concentration in Mie resonators at the subwavelength scale, much higher than usual systems. Our first experiment for third-harmonic generation was from isolated silicon (a few hundred nanometers in size) on a disk, and the resulting third harmonic was seen by naked eyes. The observed conversion efficiency can reach a few percent, which is unbelievable for nanophotonics. In contrast, the harmonic generation given by plasmonics structures is orders of magnitude weaker, and usually people do not even bother to measure it.

This high efficiency of harmonic generation from subwavelength Mie resonators has nothing to do with phase matching or quasi-phase matching; it comes from mode matching and resonances. It is better having resonances in both fundamental and higher harmonic fields. Such a high conversion completely changed the entire philosophy of nonlinear nanophotonics. Take silicon as an example: such a high level of energy already excites electrons. The resulting free electrons correspond to non-perturbative nonlinear optics with higher harmonics generated, and that is what people just started to discuss.

Besides harmonic generation, we observed stimulated Raman scattering as well. We also investigated optical lasing in Mie resonators so that we can have a subwavelength laser visible as small as 300 nanometer in size.

Guoqing Chang: Design and optimization of metamaterial structures usually involves heavy computational work. How did you design these structures to produce the results as you expect?

Yuri Kivshar: My interest is, basically, to understand and explain the properties of these systems with deep physics based on resonances and coupling effects. I am really surprised to hear that many scientists 
jumped to study machine learning and artificial intelligence. Machine learning is nothing but a very sophisticated computational algorithm. That is not my approach, and I want to understand what happens from physical insights.

Take the Kerker effect as an example: when you overlap magnetic and electric dipoles, the reflection can be eliminated by arranging the fields, and you can achieve unidirectional transmission. But this is a very special condition to overlap amplitude and frequency of these magnetic and electric dipoles. It is not broadband. We asked how to make it broadband. We increased the size of the pillars to introduce the second magnetic resonance. These two Mie resonances are quantized by Fabry-Pérot resonances. If the frequency of the input wave falls between these resonances, the transmission is substantially increased. From basic physics, we can predict what is discovered by many numerical computations.

Guoqing Chang: My background is electric engineering, and then I moved into optics. So, I care more about the applications. What technology in your research fields has a commercial potential?

Yuri Kivshar: I think metasurfaces have a great future. First, their structures are much easier to fabricate with many available technologies. Second, you have such a high level of control of every element, and you basically can do a lot of things. Metasurfaces are suggested for computation, imaging, sensing, and many other applications. Next step is to combine metasurface with two-dimensional materials to utilize various resonant effects and tunability, which has a big potential for fabricating functional devices.

Guoqing Chang: You started your career as a theorist, and now your group works on both theories and experiments. How did you make such a transition?

Yuri Kivshar: I was trained as a theorist. In the Landau School, it was tradition to pass the exams in theoretical physics to broaden the understanding of physics through theory. Before I moved to Australia, I was doing research as a theorist. When this field of photonic crystals started, it became clear that we should and can do something experimentally. And we can do experiments much easier for microwaves. I applied for a grant to study nonlinear effects in photonic crystals and invested half of the grant funds into experiments.

Of course, in every case, some efforts were required to get the right people involved. For example, I had a very good student, Ilya Shadrivov, who is now a professor in my university. Ilya Shadrivov was a theory student who was recruited from Russia to do a $\mathrm{PhD}$ with us, and he was quite good. Once he took vacation and went back to Russia. With another colleague, I entered his office looking for a book Ilya borrowed. I saw in his office a very nice coffee table made of hardwood. Australia is famous for hardwood. My colleague told me Ilya made it by himself as a hobby. When he came back from Russia, I told Ilya that he should become an experimentalist. Then he started this microwave experiment in our group with the help from others. That is how we started, and then slowly we moved from microwaves to optics.
Guoqing Chang: You collaborated with researchers all around the world. What is your view on collaboration?

Yuri Kivshar: I am open to collaborations. The most important thing in scientific collaboration is to remain honest and open. Science builds on openness in the exchange of ideas. I am glad to include collaborators in my projects, which then opens the possibility for me to participate in and contribute to their projects. Effective collaboration produces superior knowledge, which is on top of what my collaborator and I both know separately. When working together, we create something new. And that is what I call collaboration.

Guoqing Chang: In an interview posted on YouTube, you said you always work and have no spare time. Is that true?

Yuri Kivshar: That's almost true. I am working all the time because it's a lot of fun with a lot of inspiration. I really enjoy the stimulus of discussion and the exchange of ideas with my students and collaborators. Doing research is not just a routine work; almost every day there is something that I find fascinating and interesting. It is full of excitement!

Yuri Kivshar is a world leader in photonics and metamaterials recognized for his seminal contributions to nonlinear physics and nonlinear optics. He received PhD degree in 1984 in Kharkov (Ukraine), and in 1989 he left the Soviet Union to work in Spain and then later Germany, as a Humboldt Research Fellow. In 1993, Yuri Kivshar moved to Australia where he established Nonlinear Physics Center at the Australian National University. Contributions of Yuri Kivshar to nonlinear photonics and metamaterials gave a birth to a new research direction of nonlinear metamaterials. He coined the term "meta-optics" with his team designed and measured different classes of the world-first metadevices with various functionalities. Yuri Kivshar is one of the founders of an entirely new field of all-dielectric resonant metaphotonics governed by the physics of Mie resonances in dielectric nanoparticles with high refractive index that derives unique functionalities from the electric and magnetic multipolar response. Yuri Kivshar is fellow of the Australian Academy of Science, OSA, APS, SPIE, and IOP. He received many national and international awards including Pnevmatikos Prize in Nonlinear Science (Greece), Lyle Medal (Australia), Lebedev Medal (Russia), The State Prize in Science and Technology (Ukraine), Harrie Massey Medal (UK), Humboldt Research Award (Germany), and SPIE Mozi Award (USA).

Guoqing Chang graduated with both bachelor's and master's degrees from the Electronics Engineering Department of Tsinghua University. $\mathrm{He}$ obtained his $\mathrm{PhD}$ degree in electrical engineering from the Center for Ultrafast Optical Science at the University of Michigan. After staying at the University of Michigan as a postdoctoral research fellow for about one year, he joined the Research Laboratory of Electronics at Massachusetts Institute of Technology as a postdoctoral research associate. In August 2012, he moved to the Center for Free Electron Laser (CFEL) at Hamburg (Germany) as the head of the Helmholtz Young investigator group "Ultrafast Laser Optics and Coherent Microscopy" under the ultrafast optics and x-ray division. He was granted tenure in 2016. In 2017, he joined Institute of Physics, Chinese Academy of Sciences, as a professor. His current research focuses on high-power ultrafast fiber lasers, ultrafast nonlinear optics, and multi-photon microscopy for biomedical imaging. 